\title{
Risk factors of morbidity and mortality following hip fracture surgery
}

\author{
Seung Dong Kim, Sang Jin Park, Deok Hee Lee, and Dae Lim Jee \\ Department of Anesthesiology and Pain Medicine, Yeungnam University College of Medicine, Daegu, Korea
}

Background: The preoperative coexisting chronic systemic illness, delay in surgery, gender, and age were considered as risk factors for the complications after hip fracture surgery. The wider range of surgical delay and immobilityrelated pulmonary morbidity may affect postoperative complications and mortality. This study examined the risk factors for morbidity and mortality following the hip fracture surgery.

Methods: The patient data was collected retrospectively. The consecutive 506 patients with hip fracture surgery, aged 60 years or older, were included. The patients' age, gender, preexisting diseases, American Society of Anesthesiologists (ASA) classification, delay in surgical repair, duration of surgical procedure, and methods of anesthesia were noted. The thirty-day postoperative complications were reviewed, and cardiac complications, pulmonary complications, delirium, and death were recorded. The data was analyzed for postoperative complications and risk factors.

Results: Atelectasis was associated with postoperative pulmonary complications. Male gender and age $\geq 80$ years were associated with an increased incidence of postoperative delirium. ASA classification 3 was associated with death. A delay in surgery was not associated with any complications. Preexisting diseases and methods of anesthesia did not affect mortality and postoperative complications.

Conclusions: The results suggest that a delay in surgery did not affect the postoperative complications and morbidity. (Korean J Anesthesiol 2013; 64: 505-510)

Key Words: Hip fractures, Morbidity, Mortality.

Received: August 30, 2012. Revised: 1st, October 31, 2012; 2nd, November 14, 2012. Accepted: November 20, 2012.

Corresponding author: Dae Lim Jee, M.D., Department of Anesthesiology and Pain Medicine, Yeungnam University Medical Center, Daemyeong 5-dong, Nam-gu, Daegu 705-717, Korea. Tel: 82-53-620-3367, Fax: 82-53-626-5275, E-mail: adjee@medical.yeungnam.ac.kr This is a Thesis for a Master's degree.

ㄷ) This is an open-access article distributed under the terms of the Creative Commons Attribution Non-Commercial License (http:// creativecommons.org/licenses/by-nc/3.0/), which permits unrestricted non-commercial use, distribution, and reproduction in any medium, provided the original work is properly cited. 


\section{Introduction}

The proportion of the elderly population is increasing due to the improvement in the quality of life and development of medical science, and the rate of the increase is expected to accelerate. The aging population implies, from a medical point of view, an increase in the number of elderly patients.

Many clinicians have shown concern for the postoperative systemic complications of hip fracture surgery in the elderly patients. Preoperative coexisting chronic systemic illness, delays in surgery, gender, and age has been implicated. In a study of the mortality after hemiarthroplasty, it was concluded that the medical condition was the most important for survival [1]. The delay in surgery more than 48 hours of the hip fractures showed an increase in mortality within 30 days to 1 year [2]. On the other hand, there is a contradictory report showing that a delay in surgery does not affect mortality [3]. Males were reported to have higher mortality than females [4]. In contrast, a recent study reported no significant difference between males and females [5]. Old age was reported to increase mortality [6], whereas another study reported that the mortality was inversely proportional to age [7].

It appears that the contradictory conclusions from the various studies may have resulted from different models with different patient characteristics. In the present study, the delay in surgery was of a wider range compared to another study [8], as some patients were not transferred in time from the primary or secondary care clinics to the author's tertiary university hospital, while others were not in good medical condition to allow timely surgery. Patients sometimes suffer from immobility-related pulmonary complications, such as atelectasis or pneumonia before surgery. Therefore, a lengthy delay in surgery and preoperative pulmonary morbidity may change morbidity and mortality after hip fracture surgery in a manner different from that reported previously. Although morbidity, mortality, and the related risk factors after hip fracture surgery have been studied, it is little known how these factors may be influential. Therefore this study examined the risk factors for morbidity and mortality after hip fracture surgery.

\section{Materials and Methods}

The patient data was collected retrospectively after obtaining the approval of the ethics committee, and the medical charts were reviewed. The consecutive 506 patients with a hip fracture, aged 60 years or older, who had undergone surgery, from January 1, 2006 to December 31, 2010 were included. Patients with metastatic cancer or multiple injuries were excluded. The age, gender, American Society of Anesthesiologists (ASA) physical status classification, delay in surgical repair (day), duration of surgical procedure, and methods of anesthesia were noted. Concurrent medical conditions were also noted, and each was defined by the diagnosis made by the consulting physicians in the respective specialty. The patients with hypertension, diabetes mellitus, cerebrovascular accident, asthma, chronic obstructive pulmonary disease (COPD), liver cirrhosis, chronic kidney disease, Parkinson's disease, and dementia were included. Cardiac morbidity, atelectasis, pulmonary consolidation, pulmonary edema, pleural effusion, and pneumonia were recorded. A history of myocardial infarction, coronary artery bypass graft surgery, atrial fibrillation, congestive heart failure, and WolffParkinson-White syndrome were grouped together as 'cardiac morbidity,' because the proportion of patients with each condition in the sample was too small. Atelectasis, pulmonary consolidation, edema, pleural effusion, and pneumonia were also recorded. Because of the very small proportion of patients with pulmonary edema, pleural effusion, and pneumonia in the sample, these conditions were also grouped as a single variable.

The thirty-day postoperative complications were reviewed and the cardiac complications, pulmonary complications, delirium, and death were recorded. The cardiac complications included arrhythmia, myocardial infarction, and congestive heart failure. Pulmonary complications included newly developed pulmonary lesions and a deterioration of the radiologic findings after surgery. Delirium included disturbances of consciousness and changes in cognition satisfying the DSM-IV after surgery. Postoperative death included two cases of intraoperative death. Thus, 504 patients were analyzed for other postoperative complications.

To determine the rates of complications within 30 days after surgery and their risk factors, categorical or continuous data were first analyzed using a chi-square test, Fisher's exact test, and binary logistic regression analysis. A P value $<0.05$ was considered significant. Statistical analysis was performed using SPSS $^{\circledR}$ 19.0. All data is expressed in number, as the mean \pm SD.

\section{Results}

The data of 506 patients was from a 5-year period. Table 1 lists the patients' characteristics. The mean (SD) age of the patients was 77.2 years (7.4 years). Four hundred and thirty four patients (85.8\%) had concurrent medical problems. In the present study, the mean delay in surgery was 6.4 days (10 days), the case of a more than 7 days delay in surgery was $119(23.5 \%)$ patients, and 10 days delay was $79(15.7 \%)$ patients. Table 2 lists the causes of delay in surgery of more than 10 days.

The independent variables listed in Table 1 were analyzed according to each postoperative complication (Table 3) using univariate analyses, and the results were presented in Table 4. 
Table 1. Characteristics of Patients

\begin{tabular}{lc}
\hline \multicolumn{1}{c}{ Variables } & Number of patients \\
\hline Total patients & 506 \\
Sex (M/F) & $140 / 366$ \\
Age & \\
$60-69$ & 83 \\
$70-79$ & 227 \\
$\geq 80$ & 196 \\
Hypertension & 258 \\
Diabetes mellitus & 131 \\
COPD & 125 \\
Atelectasis & 123 \\
Cerebrovascular accident & 98 \\
Cardiac morbidity & 54 \\
Pulmonary consolidation & 49 \\
Dementia & 40 \\
Pulmonary edema, effusion, pneumonia & 40 \\
Chronic kidney disease & 25 \\
Asthma & 19 \\
Parkinson's disease & 16 \\
Liver cirrhosis & 6 \\
ASA (1/2/3) & $6 / 311 / 189$ \\
Anesthesia & \\
General & 246 \\
Spinal & 249 \\
Epidural & 11 \\
& Mean \pm SD \\
Delay in surgery (day) & $6.4 \pm 10$ \\
Duration of surgical procedure (min) & $75.8 \pm 29.8$ \\
V & \\
\hline & \\
\hline &
\end{tabular}

Values are number or mean \pm SD. COPD: chronic obstructive pulmonary disease, Effusion: pleural effusion, ASA: American Society of Anesthesiologists classification.

Preoperative cardiac morbidity did not increase the number of cardiac complication, whereas ASA classification 3 increased the cardiac complications. The incidence of pulmonary complications was higher in patients with atelectasis, pulmonary consolidation, or ASA Classification 3. Male gender, age $\geq 80$ years, and COPD were associated with an increased incidence of postoperative delirium. ASA Classification 3 was associated with death. A delay in surgery did not show significance with any postoperative complications.

Table 5 presents the results of binary logistic regression analysis. In this model, atelectasis was associated with postoperative pulmonary complications. Male gender and age $\geq \mathbf{8 0}$ years were associated with an increased incidence of postoperative delirium. ASA Classification 3 was associated with death.

\section{Discussion}

Many clinicians have noted the preoperative risk factors of hip fracture surgery, and the recognition of such has helped to avoid postoperative complications. This study examined whether patients with a lengthy delay in surgery and preoperative pulmonary morbidity were affected in the morbidity and
Table 2. Causes of Delay in Surgery more than 10 Days

\begin{tabular}{lc}
\hline \multicolumn{1}{c}{ Causes of delay in surgery } & Number of patients (rate) \\
\hline Total patients & 79 \\
Medical condition & $44(55.7 \%)$ \\
Conservative treatment* & $14(17.7 \%)$ \\
Delay to visit the hospital by & $10(12.7 \%)$ \\
unawareness of hip fracture & $3(3.8 \%)$ \\
Misdiagnosis & $8(10.1 \%)$ \\
Unknown cause & \\
\hline
\end{tabular}

*Conservative treatment in all care clinics including the Traditional Asian Medicine clinics.

Table 3. Types and Incidences of Postoperative Complications

\begin{tabular}{lcc}
\hline \multicolumn{2}{c}{ Type (Total number of patients) } & Number of patients (Incidence) \\
\hline Cardiac complication & $(504)$ & $6(1.2 \%)$ \\
Pulmonary complication & $(504)$ & $168(33.3 \%)$ \\
Delirium & $(504)$ & $59(11.7 \%)$ \\
Death & $(506)$ & $11(2.17 \%)$ \\
\hline
\end{tabular}

Values are number (incidence). Cardiac complication included arrhythmia, myocardial infarction, and congestive heart failure. Pulmonary complication included newly developed pulmonary lesions and deterioration of the radiologic findings after surgery. Delirium included disturbances of consciousness and changes in cognition satisfying the DSM-IV. Death included two cases of intraoperative death.

mortality after hip fracture surgery. The results showed that a lengthy delay in surgery did not increase any postoperative complications, and among the preoperative pulmonary morbidities, only atelectasis was found to increase the number of postoperative pulmonary complication. Preoperative cardiac or other morbidities, such as hypertension, COPD, pulmonary consolidation, or diabetes mellitus and dementia, did not affect the postoperative outcomes in hip fracture surgery.

The thirty day mortality after hip fracture surgery was reported to be $5-10 \%$ [9], which was higher than that reported in the present study $(2.17 \%)$. The reason for the discrepancy in mortality is unclear. However, it was also reported that the mortality rate after hip fracture surgery was 3.5\% [6], which was similar to that reported in the present study. Advanced age has been considered as a risk factor of mortality, but there is a controversy. Carretta et al. [6] reported that advanced age was a risk factor for mortality after hip fracture surgery. There was an inverse relationship between mortality and advanced age in another study [7]. The results in the present study demonstrated that advanced age was not associated with mortality. In contrast, mortality was higher in patients with ASA Classification 3, including two cases of intraoperative death. This suggests that the preoperative patient's condition is important for survival, as the ASA classification reflects the general condition of the patient. Furthermore, advanced age and poor general condition are not always in accord. Therefore, a high ASA 
Table 4. Univariate Analysis of Potential Risk Factors

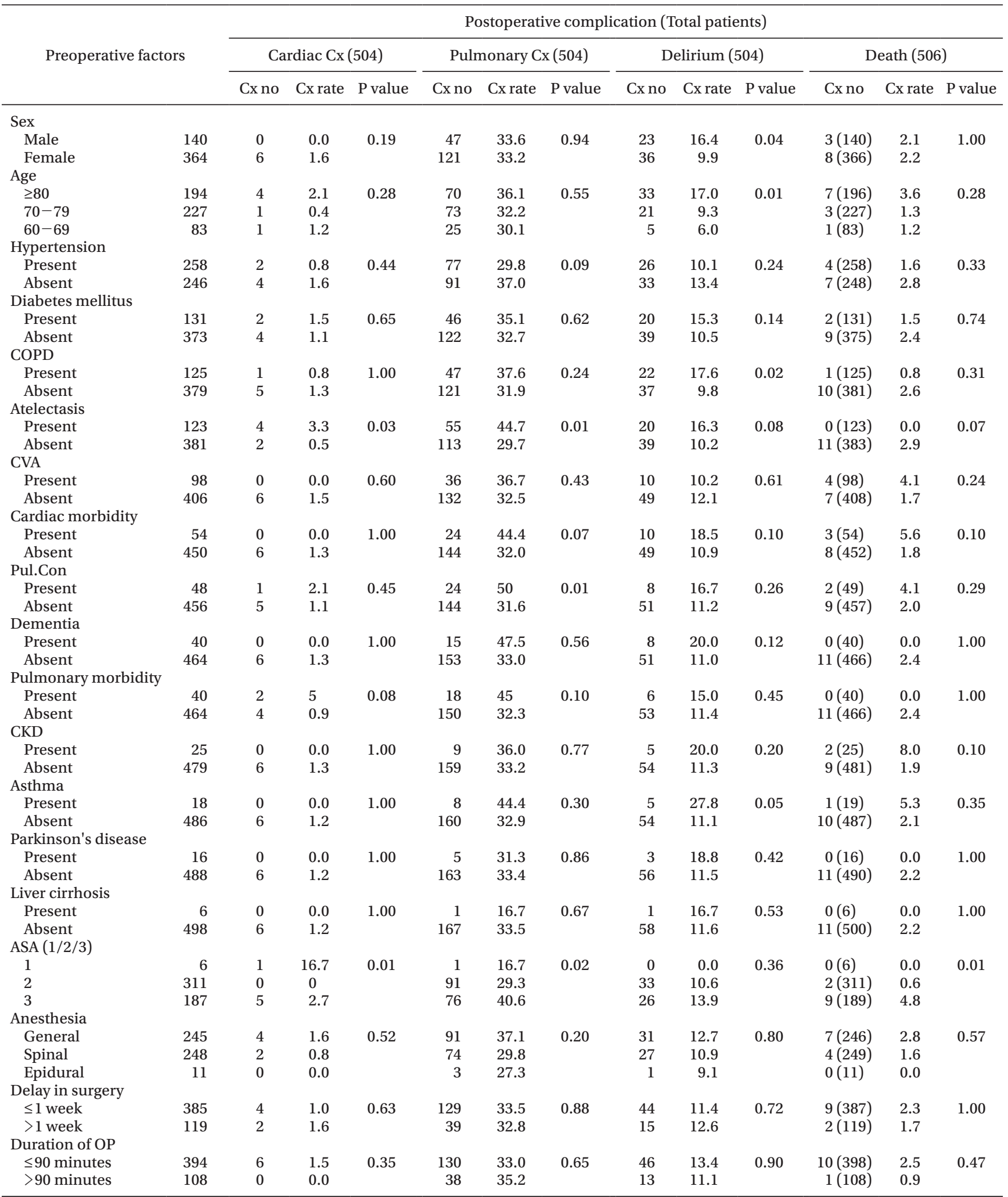

$\mathrm{Cx}$ : complication, Cardiac Cx: arrythmia, myocardiac infarction, congestive heart failure, Pulmonary Cx: newly developed pulmonary lesion, deterioration of the radiologic finding, CVA: cerebrovascular accident, COPD: chronic obstructive pulmonary disease, CKD: chronic kidney disease, Pul.Con: pulmonary consolidation, Pulmonary morbidity: pulmonary edema, pleural effusion, pneumonia, ASA: American Society of Anesthesiologists classification, Duration of OP: Duration of surgical procedure. 
Table 5. Binary Logistic Regression Model

\begin{tabular}{|c|c|c|c|}
\hline & Odds ratio & $\begin{array}{l}\text { 95\% Confidence } \\
\text { interval }\end{array}$ & $P$ value \\
\hline Atelectasis & $\begin{array}{l}\text { Pulmonary complication } \\
1.92 \\
\text { Delirium }\end{array}$ & $1.26-2.91$ & 0.02 \\
\hline Sex (male) & 2.26 & $1.25-4.09$ & 0.01 \\
\hline Age $(\geq 80)$ & $\begin{array}{l}2.19 \\
\text { Death }\end{array}$ & $1.41-3.41$ & 0.01 \\
\hline ASA 3 & 7.77 & $1.17-36.18$ & 0.01 \\
\hline
\end{tabular}

ASA: American Society of Anesthesiologists classification.

classification level appears to be more important than age. One study reported the incidence of symptomatic venous thromboembolism of $4.4 \%$ following hip fracture surgery in the Republic of Korea, and noted on the ineffectiveness of antiplatelet [10]. Thromboembolism was considered fatal complication generally. Other studies supporting early surgery reported that the risks of postoperative complication, including serious bacterial infection, thromboembolism, and death were decreased by reducing the periods of immobility $[11,12]$. There were also studies which reported that a delay in surgery more than one week was related to higher mortality [13], and that surgery performed within 14-72 hours could reduce such [14]. However, others have reported that there was no relationship between a delay in surgery and mortality [15]. Based on the present results that a delay in surgery has not increased postoperative complications, the consideration of delaying the hip fracture surgery upon the poor patient medical condition may be recommended.

According to one report, $55.8 \%$ of patients with a hip fracture had a delay in surgery of more than 24 hours, and most causes of delay were medical problems and medical investigations [16]. In the present study, the case of a more than 7 days delay in surgery was 119 (23.5\%) patients, and 10 days delay was 79 (15.7\%) patients. The causes of the delay were the patients' being unaware of the hip fracture, conservative treatment, misdiagnosis of the fracture, poor medical condition, and preference for Traditional Asian Medicine, including acupuncture, moxa cautery, and physical therapy. The most common cause of delay in surgery was the patient's medical condition, which was in accordance with a previous study [16].

The rate of postoperative cardiac complications after hip fracture surgery was $3.6-8 \%[17,18]$. In the present study, the rate of postoperative cardiac complications was $1.2 \%$. The reason for the difference in incidence between the previous studies and the present study may be in the racial differences, such as the Western patients having a higher incidence of cardiac morbidity than the Korean patients. Postoperative pulmonary complications are a major cause of mortality after hip fracture surgery [11]. The rate of pulmonary complications after a surgery for hip fracture was $4 \%$ [17], whereas it was $33.3 \%$ in the present study. The reason for the high frequency of postoperative pulmonary complications may be due to the high incidence $(24.3 \%, 123$ patients) of preoperative atelectasis in the present results. Atelectasis is believed to be a consequence of fracture related immobility [19]. In this report, the mean duration of operative delay was longer than the mean preoperative length of stay at 3.3 days in another research [15]; and the incidence of atelectasis may have been increased by prolonged immobility.

The rate of delirium after hip fracture surgery was 30.2$36.4 \%[20,21]$. Delirium was associated with advanced age [22], dementia, anticholinergic drugs, neuroleptic drugs [23], and alcohol abuse [24]. In the present study, the rate of postoperative delirium was $11.7 \%$ and it was associated with male gender and advanced age. The effect of advanced age was in accordance with earlier reports [22]. On the other hand, preoperative dementia did not affect postoperative delirium in the present study. The reason for this is unclear. A specialized geriatric fracture center reported a lower incidence of postoperative delirium despite the higher rate of dementia [25], suggesting that dementia might not increase the incidence of postoperative delirium. Further study is needed concerning this. Male gender was reported to be a risk factor for delirium [26], which was in accordance with the present study, and the alcohol abuse was noted as a possible factor [27].

Regional anesthesia was reported to be associated with a decreased mortality compared to general anesthesia [5]. On the other hand, Heidari et al. [28] reported no relationship between mortality and the methods of anesthesia. In the present study, the method of anesthesia was not associated with postoperative complications. Anesthesia may not be an important factor in the postoperative outcomes. It seems that a short surgical procedure and moderate stress may play a role.

This study has several limitations. As a retrospective study, there were many uncontrolled variables. The decision for medical investigation before hip fracture surgery was settled by diverse clinicians. Furthermore, many prophylactic procedures like stocking for thromboembolism or lung expansion technique for pulmonary complication were not included, because of the discrepancy between the doctor's orders and practice from the difficulties with expense. Those techniques could have affected the results. In conclusion, a delay in surgery did not deteriorate the postoperative outcomes, and atelectasis increased the incidence of postoperative pulmonary complications. ASA Classification 3 was associated with mortality. Male gender and advanced age were associated with an increased incidence of postoperative delirium. The results suggest that a delay in surgery does not affect the postoperative complications and morbidity. 


\section{References}

1. Eiskjaer S, Ostgård SE. Risk factors influencing mortality after bipolar hemiarthroplasty in the treatment of fracture of the femoral neck. Clin Orthop Relat Res 1991; 270: 295-300.

2. Shiga T, Wajima Z, Ohe Y. Is operative delay associated with increased mortality of hip fracture patients? Systematic review, meta-analysis, and meta-regression. Can J Anaesth 2008; 55: 146-54.

3. Stoddart J, Horne G, Devane P. Influence of preoperative medical status and delay to surgery on death following a hip fracture. ANZ J Surg 2002; 72: 405-7.

4. Dahl E. Mortality and life expectancy after hip fractures. Acta Orthop Scand 1980; 51: 163-70.

5. Kesmezacar H, Ayhan E, Unlu MC, Seker A, Karaca S. Predictors of mortality in elderly patients with an intertrochanteric or a femoral neck fracture. J Trauma 2010; 68: 153-8.

6. Carretta E, Bochicchio V, Rucci P, Fabbri G, Laus M, Fantini MP. Hip fracture: effectiveness of early surgery to prevent 30-day mortality. Int Orthop 2011; 35: 419-24.

7. White BL, Fisher WD, Laurin CA. Rate of mortality for elderly patients after fracture of the hip in the 1980's. J Bone Joint Surg Am 1987; 69: 1335-40.

8. Moran CG, Wenn RT, Sikand M, Taylor AM. Early mortality after hip fracture: is delay before surgery important? J Bone Joint Surg Am 2005; 87: 483-9.

9. Parker M, Johansen A. Hip fracture. BMJ 2006; 333: 27-30.

10. Ji HM, Lee YK, Ha YC, Kim KC, Koo KH. Little impact of antiplatelet agents on venous thromboembolism after hip fracture surgery. J Korean Med Sci 2011; 26: 1625-9.

11. Perez JV, Warwick DJ, Case CP, Bannister GC. Death after proximal femoral fracture-an autopsy study. Injury 1995; 26: 237-40.

12. Hamlet WP, Lieberman JR, Freedman EL, Dorey FJ, Fletcher A, Johnson EE. Influence of health status and the timing of surgery on mortality in hip fracture patients. Am J Orthop (Belle Mead NJ) 1997; 26: 621-7.

13. Bae IT, Lee WS, Kim SB, Heo YM, Park CY. Postoperative complications of hip fractures in patients with chronic kidney disease. J Korean Hip Soc 2009; 21: 169-73.

14. Simunovic N, Devereaux PJ, Sprague S, Guyatt GH, Schemitsch E, Debeer J, et al. Effect of early surgery after hip fracture on mortality and complications: systematic review and meta-analysis. CMAJ 2010; 182: 1609-16.
15. Rae HC, Harris IA, McEvoy L, Todorova T. Delay to surgery and mortality after hip fracture. ANZ J Surg 2007; 77: 889-91.

16. Charalambous CP, Yarwood S, Paschalides C, Siddique I, Hirst P, Paul A. Factors delaying surgical treatment of hip fractures in elderly patients. Ann R Coll Surg Engl 2003; 85: 117-9.

17. Lawrence VA, Hilsenbeck SG, Noveck H, Poses RM, Carson JL. Medical complications and outcomes after hip fracture repair. Arch Intern Med 2002; 162: 2053-7.

18. Smektala R, Endres HG, Dasch B, Maier C, Trampisch HJ, Bonnaire F, et al. The effect of time-to-surgery on outcome in elderly patients with proximal femoral fractures. BMC Musculoskelet Disord 2008; 9: 171.

19. Goldhill DR, Imhoff M, McLean B, Waldmann C. Rotational bed therapy to prevent and treat respiratory complications: a review and meta-analysis. Am J Crit Care 2007; 16: 50-61.

20. Juliebø V, Bjøro K, Krogseth M, Skovlund E, Ranhoff AH, Wyller TB. Risk factors for preoperative and postoperative delirium in elderly patients with hip fracture. J Am Geriatr Soc 2009; 57: 1354-61.

21. Lee KH, Ha YC, Lee YK, Kang H, Koo KH. Fequency, risk factors, and prognosis of prolonged delirium in elderly patients after hip fracture surgery. Clin Orthop Relat Res 2011; 469: 2612-20.

22. Leentjens AF, van der Mast RC. Delirium in elderly people: an update. Curr Opin Psychiatry 2005; 18: 325-30.

23. Bitsch M, Foss N, Kristensen B, Kehlet H. Pathogenesis of and management strategies for postoperative delirium after hip fracture a review. Acta Orthop Scand 2004; 75: 378-89.

24. Inouye SK. Delirium in older persons. N Engl J Med 2006; 354: 115765.

25. Friedman SM, Mendelson DA, Bingham KW, Kates SL. Impact of a comanaged Geriatric Fracture Center on short-term hip fracture outcomes. Arch Intern Med 2009; 169: 1712-7.

26. Fisher BW, Flowerdew G. A simple model for predicting postoperative delirium in older patients undergoing elective orthopedic surgery. J Am Geriatr Soc 1995; 43: 175-8.

27. Tabet N, Stewart R, Hudson S, Sweeney V, Sauer J, Bryant C, et al. Male gender influences response to an educational package for delirium prevention among older people: a stratified analysis. Int J Geriatr Psychiatry 2006; 21: 493-7.

28. Heidari SM, Soltani H, Hashemi SJ, Talakoub R, Soleimani B. Comparative study of two anesthesia methods according to postoperative complications and one month mortality rate in candidates of hip surgery. J Res Med Sci 2011; 16: 323-30. 